Supporting information for

\title{
Ionic Dynamics of Hydroxylammonium Ionic Liquids: A Classical Molecular Dynamics Simulation Study
}

\section{Th. Dhileep N. Reddy and Bhabani S. Mallik}

Department of Chemistry, Indian Institute of Technology Hyderabad, Kandi-502285, Sangareddy, Telangana, India *bhabani@iith.ac.in

Table S1: Non-bonding force field parameters of cations used in the study.

\begin{tabular}{|c|c|c|c|c|}
\hline System & $\begin{array}{l}\text { Atom } \\
\text { (atom } \\
\text { type) }\end{array}$ & $q(e)$ & $\sigma(\AA)$ & $\begin{array}{c}\varepsilon \\
(\mathrm{kcal} / \mathrm{mol})\end{array}$ \\
\hline \multirow[t]{12}{*}{ HEA } & N1(n4) & -0.5310 & 3.25 & 0.1700 \\
\hline & H3(hn) & 0.3287 & 1.07 & 0.0157 \\
\hline & H4(hn) & 0.3170 & 1.07 & 0.0157 \\
\hline & H7(hn) & 0.3170 & 1.07 & 0.0157 \\
\hline & $\mathrm{C} 1(\mathrm{c} 3)$ & 0.1313 & 3.40 & 0.1094 \\
\hline & H1(hx) & 0.0896 & 1.96 & 0.0157 \\
\hline & H2(hx) & 0.0896 & 1.96 & 0.0157 \\
\hline & $\mathrm{C} 2(\mathrm{c} 3)$ & 0.1207 & 3.40 & 0.1094 \\
\hline & H5(h1) & 0.0357 & 2.47 & 0.0157 \\
\hline & H6(h1) & 0.0357 & 2.47 & 0.0157 \\
\hline & O1(oh) & -0.5054 & 3.07 & 0.2104 \\
\hline & H8(ho) & 0.3712 & 0.00 & 0.0000 \\
\hline \multirow[t]{10}{*}{ DHEA } & O1(oh) & -0.5054 & 3.07 & 0.2104 \\
\hline & H7(ho) & 0.3633 & 3.07 & 0.2104 \\
\hline & $\mathrm{C} 2(\mathrm{c} 3)$ & 0.1446 & 3.40 & 0.1094 \\
\hline & H5(h1) & 0.0238 & 2.47 & 0.0157 \\
\hline & H6(h1) & 0.0238 & 2.47 & 0.0157 \\
\hline & $\mathrm{C} 1(\mathrm{c} 3)$ & 0.1176 & 3.40 & 0.1094 \\
\hline & H3(hx) & 0.0754 & 1.96 & 0.0157 \\
\hline & H4(hx) & 0.0754 & 1.96 & 0.0157 \\
\hline & N1(n4) & -0.352 & 3.25 & 0.1700 \\
\hline & H1(hn) & 0.2566 & 1.07 & 0.0157 \\
\hline
\end{tabular}




\begin{tabular}{|c|c|c|c|c|}
\hline & H2(hn) & 0.2566 & 1.07 & 0.0157 \\
\hline & C3(c3) & 0.1176 & 3.40 & 0.1094 \\
\hline & H8(hx) & 0.0755 & 1.96 & 0.0157 \\
\hline & H9(hx) & 0.0755 & 1.96 & 0.0157 \\
\hline & $\mathrm{C} 4(\mathrm{c} 3)$ & 0.1477 & 3.40 & 0.1094 \\
\hline & H10(h1) & 0.0227 & 2.47 & 0.0157 \\
\hline & H11(h1) & 0.0227 & 2.47 & 0.0157 \\
\hline & $\mathrm{O} 2(\mathrm{oh})$ & -0.5058 & 3.07 & 0.2104 \\
\hline & H12(oh) & 0.3643 & 0.00 & 0.0000 \\
\hline \multirow[t]{26}{*}{ THEA } & O1(oh) & -0.5377 & 3.07 & 0.2104 \\
\hline & H14(ho) & 0.3684 & 0.00 & 0.0000 \\
\hline & $\mathrm{C} 4(\mathrm{c} 3)$ & 0.1278 & 3.40 & 0.1094 \\
\hline & H8(h1) & 0.0731 & 2.47 & 0.0157 \\
\hline & H9(h1) & 0.0227 & 2.47 & 0.0157 \\
\hline & $\mathrm{C} 2(\mathrm{c} 3)$ & -0.1353 & 3.40 & 0.1094 \\
\hline & H4(hx) & 0.0828 & 1.96 & 0.0157 \\
\hline & H5(hx) & 0.1304 & 1.96 & 0.0157 \\
\hline & N1(n4) & 0.0546 & 3.25 & 0.1700 \\
\hline & H1(hn) & 0.2018 & 1.07 & 0.0157 \\
\hline & C3(c3) & -0.01490 & 3.40 & 0.1094 \\
\hline & H6(hx) & 0.1105 & 1.96 & 0.0157 \\
\hline & H7(hx) & 0.1117 & 1.96 & 0.0157 \\
\hline & C6(c3) & 0.0685 & 3.40 & 0.1094 \\
\hline & H12(h1) & 0.0588 & 2.47 & 0.0157 \\
\hline & H13(h1) & 0.0512 & 2.47 & 0.0157 \\
\hline & O3(oh) & -0.5046 & 3.07 & 0.2104 \\
\hline & H16(ho) & 0.3610 & 0.00 & 0.0000 \\
\hline & $\mathrm{C} 1(\mathrm{c} 3)$ & -0.1166 & 3.40 & 0.1094 \\
\hline & H2(hx) & 0.0991 & 1.96 & 0.0157 \\
\hline & H3(hx) & 0.1451 & 1.96 & 0.0157 \\
\hline & $\mathrm{C} 5(\mathrm{c} 3)$ & 0.0874 & 3.40 & 0.1094 \\
\hline & H10(h1) & 0.0865 & 2.47 & 0.0157 \\
\hline & H11(h1) & 0.0226 & 2.47 & 0.0157 \\
\hline & $\mathrm{O} 2(\mathrm{oh})$ & -0.5267 & 3.07 & 0.2104 \\
\hline & H15(ho) & 0.3717 & 0.00 & 0.0000 \\
\hline
\end{tabular}


Table S2: Non-bonding force field parameters of anions used in the study.

\begin{tabular}{|c|c|c|c|c|}
\hline System & $\begin{array}{c}\text { Atom(Atom } \\
\text { type) }\end{array}$ & $\mathrm{q}(\mathrm{e})$ & $\sigma(\AA)$ & $\varepsilon(\mathrm{kcal} / \mathrm{mol})$ \\
\hline FMT & O1(o) & -0.6522 & 2.96 & 0.2100 \\
\hline & C1(c) & 0.6678 & 3.40 & 0.0860 \\
\hline & H1(h5) & -0.1634 & 2.42 & 0.015 \\
\hline & O2(o) & -0.6522 & 2.96 & 0.2100 \\
\hline LAC & O1(o) & -0.6107 & 2.96 & 0.2100 \\
\hline & C1(c) & 0.5251 & 3.40 & 0.0860 \\
\hline & O2(o) & -0.5986 & 2.96 & 0.2100 \\
\hline & C2(c3) & 0.4297 & 3.40 & 0.1094 \\
\hline & H4(h1) & -0.0880 & 2.47 & 0.0157 \\
\hline & O3(oh) & -0.6201 & 3.07 & 0.2104 \\
\hline & H5(ho) & 0.2879 & 0.00 & 0.0000 \\
\hline & C3(c3) & -0.1624 & 3.40 & 0.1094 \\
\hline & H1(hc) & 0.0026 & 2.65 & 0.0157 \\
\hline & H2(hc) & 0.0145 & 2.65 & 0.0157 \\
\hline & H3(hc) & 0.0200 & 2.65 & 0.0157 \\
\hline
\end{tabular}

Table S3: Densities of ionic liquids used in this study and the literature.

\begin{tabular}{|c|c|c|c|c|}
\hline \multirow{2}{*}{} & \multicolumn{2}{|c|}{ Density $\left({\left.\mathrm{g} . \mathrm{cm}^{-3}\right) \text { at } 298.15 \mathrm{~K}}^{2}\right.$ Density $\left(\mathrm{g} . \mathrm{cm}^{-3}\right)$ at $338.15 \mathrm{~K}$} \\
\hline IL & Our Work & Literature & Our Work & Literature \\
\hline HEF & 1.284 & $1.204^{1-5}, 1.176^{6}, 1.184^{7,8}, 1.140^{9}$, & 1.259 & $1.148^{6}, 1.115^{9}$ \\
\hline DHEF & 1.283 & $1.194^{6}, 1.039^{8}, 1.219^{9}$ & 1.256 & $1.196^{9}$ \\
\hline THEF & 1.301 & - & 1.276 & $1.207^{9}, 1.213^{6}$ \\
\hline HEL & 1.245 & $1.205^{5}, 1.228^{7}, 1.202^{5}$ & 1.217 & - \\
\hline
\end{tabular}




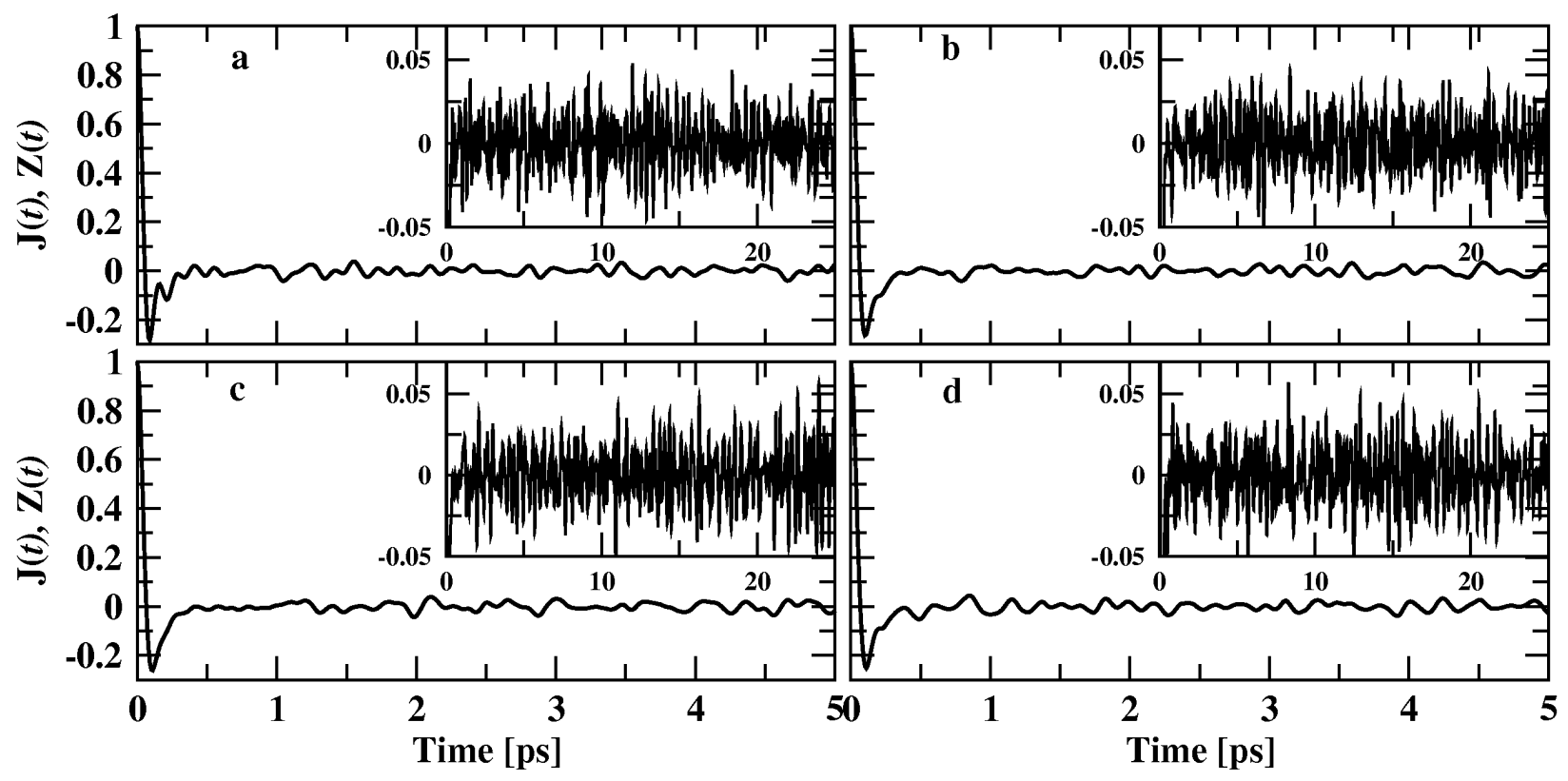

Figure S1: Current autocorrelation functions (CACFs) of HEF, DHEF, THEF and HEL are shown in a, b, c and d respectively. The long time behavior of CACFs is shown in insets.

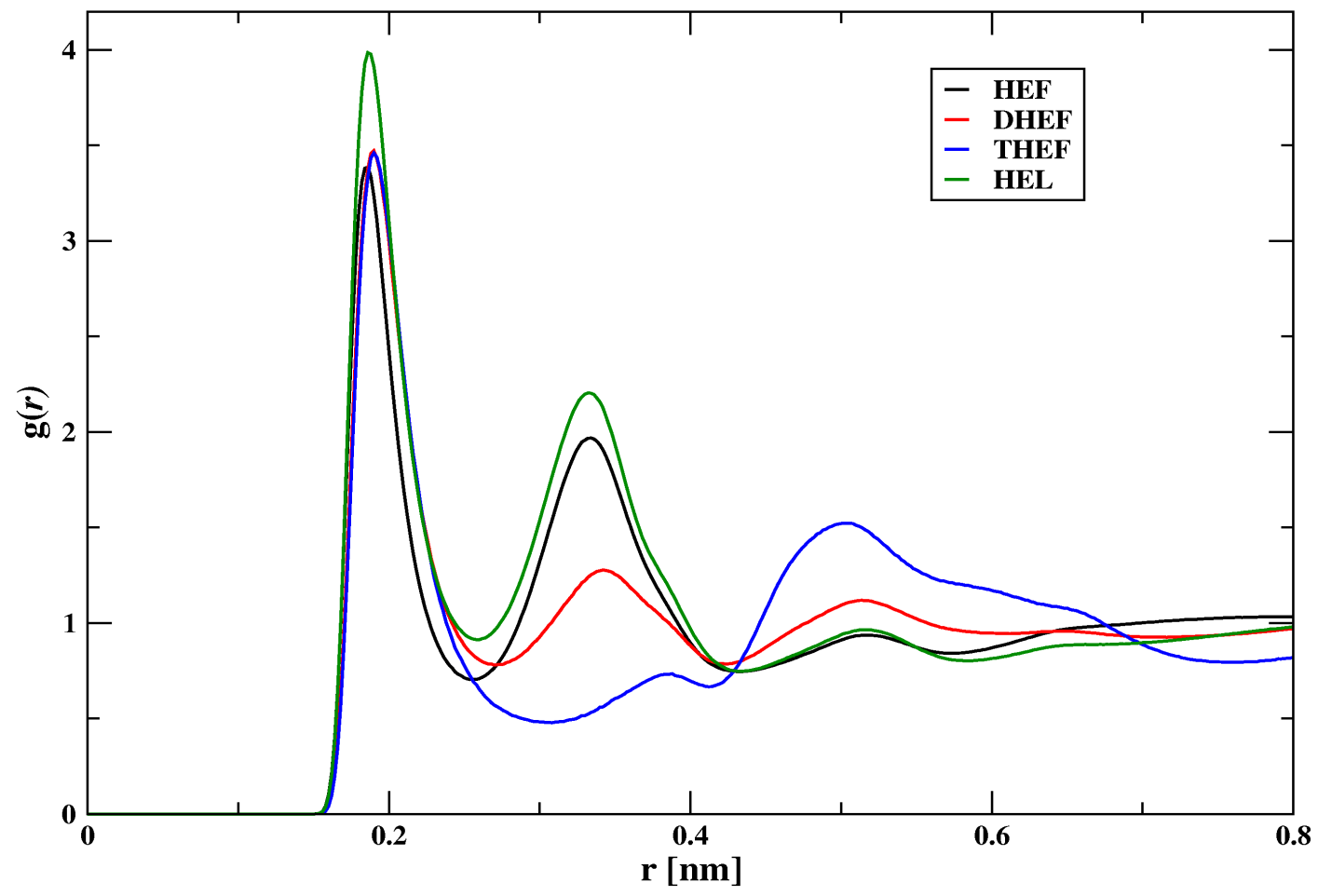

Figure S2: Radial distribution functions between ammonuium hydrogen atom of cation $\left(\mathrm{H}_{\mathrm{N}}\right)$ and carboxylate oxygen atoms of anion $\left(\mathrm{Oc}_{\mathrm{c}}\right)$. 
XYZ files of optimized ions in this study.

Hydroxyethylammonium cation (HEA)

$\begin{array}{lrrr}12 & & & \\ \mathrm{~N} & -1.474000 & 1.059000 & 0.000000 \\ \mathrm{H} & -1.602000 & 2.075000 & 0.000000 \\ \mathrm{H} & -1.952000 & 0.687000 & -0.827000 \\ \mathrm{H} & -1.952000 & 0.687000 & 0.827000 \\ \mathrm{C} & 0.000000 & 0.693000 & 0.000000 \\ \mathrm{H} & 0.442000 & 1.141000 & 0.888000 \\ \mathrm{H} & 0.442000 & 1.141000 & -0.888000 \\ \mathrm{C} & 0.182000 & -0.821000 & 0.000000 \\ \mathrm{H} & -0.288000 & -1.260000 & 0.891000 \\ \mathrm{H} & -0.288000 & -1.260000 & -0.891000 \\ \mathrm{O} & 1.577000 & -0.991000 & 0.000000 \\ \mathrm{H} & 1.806000 & -1.928000 & 0.000000\end{array}$

Dihydroxyethylammonium cation (DHEA)

19

$\begin{array}{llll}\text { O } & 3.583000 & -0.414000 & -0.000000\end{array}$

$\begin{array}{llll}\mathrm{H} & 4.423000 & 0.060000 & -0.000000\end{array}$

$\begin{array}{llll}\text { C } & 2.505000 & 0.491000 & -0.000000\end{array}$

$\begin{array}{llll}\mathrm{H} & 2.511000 \quad 1.135000 & -0.891000\end{array}$

$\begin{array}{llll}\mathrm{H} & 2.511000 & 1.135000 & 0.891000\end{array}$

$\begin{array}{llll}\text { C } & 1.268000 & -0.401000 & -0.000000\end{array}$

$\mathrm{H} \quad 1.246000 \quad-1.033000 \quad-0.887000$

$\begin{array}{llll}\mathrm{H} & 1.246000 & -1.033000 & 0.886000\end{array}$

$\begin{array}{llll}\mathrm{N} & 0.000000 & 0.420000 & 0.000000\end{array}$

$\begin{array}{llll}\mathrm{H} & 0.001000 & 1.037000 & 0.818000\end{array}$

$\begin{array}{llll}\mathrm{H} & 0.001000 & 1.037000 & -0.817000\end{array}$ 


$\begin{array}{lrrr}\mathrm{C} & -1.268000 & -0.400000 & -0.000000 \\ \mathrm{H} & -1.247000 & -1.032000 & 0.886000 \\ \mathrm{H} & -1.247000 & -1.032000 & -0.886000 \\ \mathrm{C} & -2.506000 & 0.491000 & 0.000000 \\ \mathrm{H} & -2.512000 & 1.135000 & -0.890000 \\ \mathrm{H} & -2.512000 & 1.135000 & 0.891000 \\ \mathrm{O} & -3.582000 & -0.414000 & 0.000000 \\ \mathrm{H} & -4.423000 & 0.058000 & 0.000000\end{array}$

trihydroxyethylammonium cation (THEA)

$\begin{array}{lrrr}26 & & & \\ \mathrm{O} & -2.615000 & -0.548000 & -0.988000 \\ \mathrm{H} & -3.299000 & -1.056000 & -1.439000 \\ \mathrm{C} & -2.444000 & -1.034000 & 0.334000 \\ \mathrm{H} & -3.390000 & -1.056000 & 0.887000 \\ \mathrm{H} & -2.040000 & -2.051000 & 0.335000 \\ \mathrm{C} & -1.532000 & -0.082000 & 1.082000 \\ \mathrm{H} & -1.325000 & -0.479000 & 2.076000 \\ \mathrm{H} & -1.992000 & 0.900000 & 1.187000 \\ \mathrm{~N} & -0.180000 & 0.159000 & 0.422000 \\ \mathrm{H} & 0.356000 & 0.695000 & 1.119000 \\ \mathrm{C} & 0.550000 & -1.131000 & 0.155000 \\ \mathrm{H} & 0.437000 & -1.756000 & 1.041000 \\ \mathrm{H} & 0.062000 & -1.622000 & -0.684000 \\ \mathrm{C} & 2.043000 & -0.961000 & -0.114000 \\ \mathrm{H} & 2.220000 & -0.496000 & -1.089000 \\ \mathrm{H} & 2.502000 & -0.333000 & 0.662000 \\ \mathrm{O} & 2.544000 & -2.281000 & -0.076000 \\ \mathrm{H} & 3.466000 & -2.296000 & -0.357000 \\ \mathrm{C} & -0.247000 & 1.111000 & -0.769000\end{array}$




$\begin{array}{lrrr}\mathrm{H} & -0.198000 & 0.531000 & -1.686000 \\ \mathrm{H} & -1.217000 & 1.597000 & -0.727000 \\ \mathrm{C} & 0.863000 & 2.149000 & -0.666000 \\ \mathrm{H} & 0.629000 & 2.965000 & -1.354000 \\ \mathrm{H} & 1.839000 & 1.739000 & -0.938000 \\ \mathrm{O} & 0.858000 & 2.582000 & 0.694000 \\ \mathrm{H} & 1.518000 & 3.270000 & 0.839000\end{array}$

Formate anion (FMT)

4

$\begin{array}{llll}\mathrm{O} & -0.000000 & 1.136000 & -0.209000 \\ \mathrm{C} & 0.000000 & -0.000000 & 0.316000 \\ \mathrm{H} & 0.000000 & -0.000000 & 1.452000 \\ \mathrm{O} & -0.000000 & -1.136000 & -0.209000\end{array}$

Lactate anion (LAC)

11

$\begin{array}{lrrr}\mathrm{O} & -1.475000 & -1.146000 & -0.220000 \\ \mathrm{C} & -0.878000 & -0.068000 & -0.039000 \\ \mathrm{O} & -1.325000 & 1.028000 & 0.404000 \\ \mathrm{C} & 0.641000 & -0.028000 & -0.409000 \\ \mathrm{H} & 0.719000 & -0.265000 & -1.479000 \\ \mathrm{O} & 1.144000 & 1.288000 & -0.187000 \\ \mathrm{H} & 0.327000 & 1.727000 & 0.149000 \\ \mathrm{C} & 1.448000 & -1.053000 & 0.382000 \\ \mathrm{H} & 1.057000 & -2.056000 & 0.201000 \\ \mathrm{H} & 2.505000 & -1.019000 & 0.096000 \\ \mathrm{H} & 1.379000 & -0.840000 & 1.453000\end{array}$


mdp files for GROMACS used in this study

\section{energy minimization}

;em.mdp - used as input into grompp to generate em.tpr

; Parameters describing what to do, when to stop and what to save

$\begin{array}{lll}\text { integrator } & =\text { steep } & \text {; Algorithm (steep = steepest descent minimization) } \\ \text { emtol } & =1.0 & ; \text { Stop minimization when the maximum force }<1000.0 \mathrm{~kJ} / \mathrm{mol} / \mathrm{nm} \\ \text { emstep } & 0.01 \quad ; \text { Energy step size } \\ \text { nsteps } & =100000 \quad ; \text { Maximum number of (minimization) steps to perform }\end{array}$

; Parameters describing how to find the neighbors of each atom and how to calculate the interactions

nstlist $\quad=40 \quad$; Frequency to update the neighbor list and long range forces

verlet-buffer-tolerance $=-1$

vdw_type $\quad=$ shift

coulombtype = PME-Switch

rcoulomb_switch $=1.15$

rvdw-switch $=1.15$

cutoff-scheme $=$ Verlet

ns_type $\quad$ grid $\quad$; Method to determine neighbor list (simple, grid)

rlist $\quad=1.3 \quad$; Cut-off for making neighbor list (short range forces)

rcoulomb $\quad=1.2 \quad$; Short-range electrostatic cut-off

rvdw $\quad=1.2 \quad$; Short-range Van der Waals cut-off

$\mathrm{pbc} \quad=\mathrm{xyz} \quad ;$ Periodic Boundary Conditions (yes/no)

\section{Heating}

; Run parameters

integrator $\quad$ md leap-frog integrator

nsteps $\quad=10000000$

dt $\quad=0.002 \quad ; 2 \mathrm{fs}$

; Output control

nstxout $\quad=3000$ 


$\begin{array}{ll}\text { nstvout } & =3000 \\ \text { nstenergy } & =3000 \\ \text { nstlog } & =3000\end{array}$

; Bond parameters

$\begin{array}{lll}\text { continuation } \quad=\text { no } & ; \text { first dynamics run } \\ \text { constraint_algorithm }=\text { lincs } & ; \text { holonomic constraints } \\ \text { constraints } \quad=\text { all-bonds } & ; \text { all bonds (even heavy atom-H bonds) constrained } \\ \text { lincs_iter } \quad=1 & ; \text { accuracy of LINCS } \\ \text { lincs_order } \quad=4 & \text {; also related to accuracy }\end{array}$

; Neighborsearching

cutoff-scheme $=$ Verlet

ns_type $\quad$ grid $\quad$; search neighboring grid cells

nstlist $\quad=40 \quad ; 10 \mathrm{fs}$

rlist $\quad=1.3 \quad$; short-range neighborlist cutoff (in $\mathrm{nm}$ )

rcoulomb $\quad=1.2 \quad$; short-range electrostatic cutoff (in nm)

rvdw $\quad=1.2 \quad ;$ short-range van der Waals cutoff (in nm)

vdw_type $\quad=$ shift

coulombtype = PME-Switch

rcoulomb_switch $=1.15$

rvdw-switch $=1.15$

verlet-buffer-tolerance $=-1$

; Electrostatics

pme_order $=4 \quad$; cubic interpolation

fourierspacing $=0.16 \quad$; grid spacing for FFT

; Temperature coupling is on

tcoupl $\quad=$ V-rescale $;$ modified Berendsen thermostat

tc-grps $\quad=$ system $\quad$; two coupling groups - more accurate

tau_t $\quad=0.1 \quad$; time constant, in ps

ref $\mathrm{t} \quad=538.15 \quad$; reference temperature, one for each group, in $\mathrm{K}$

; Pressure coupling is off 
pcoupl $\quad=$ no $\quad$ no pressure coupling in NVT

; Periodic boundary conditions

$\mathrm{pbc} \quad=\mathrm{xyz} \quad ; 3-\mathrm{D}$ PBC

; Dispersion correction

\section{Annealing}

PLS Lysozyme NVT equilibration

define $\quad=$-DPOSRES ; position restrain the protein

; Run parameters

$\begin{array}{lll}\text { integrator } & =\mathrm{sd} & \\ \mathrm{nsteps} & =500000 & \\ \mathrm{dt} & =0.002 & ; 2 \mathrm{fs}\end{array}$

; Output control

$\begin{array}{ll}\text { nstxout } & =3000 \\ \text { nstvout } & =3000 \\ \text { nstenergy } & =3000 \\ \text { nstlog } & =3000\end{array}$

; Bond parameters

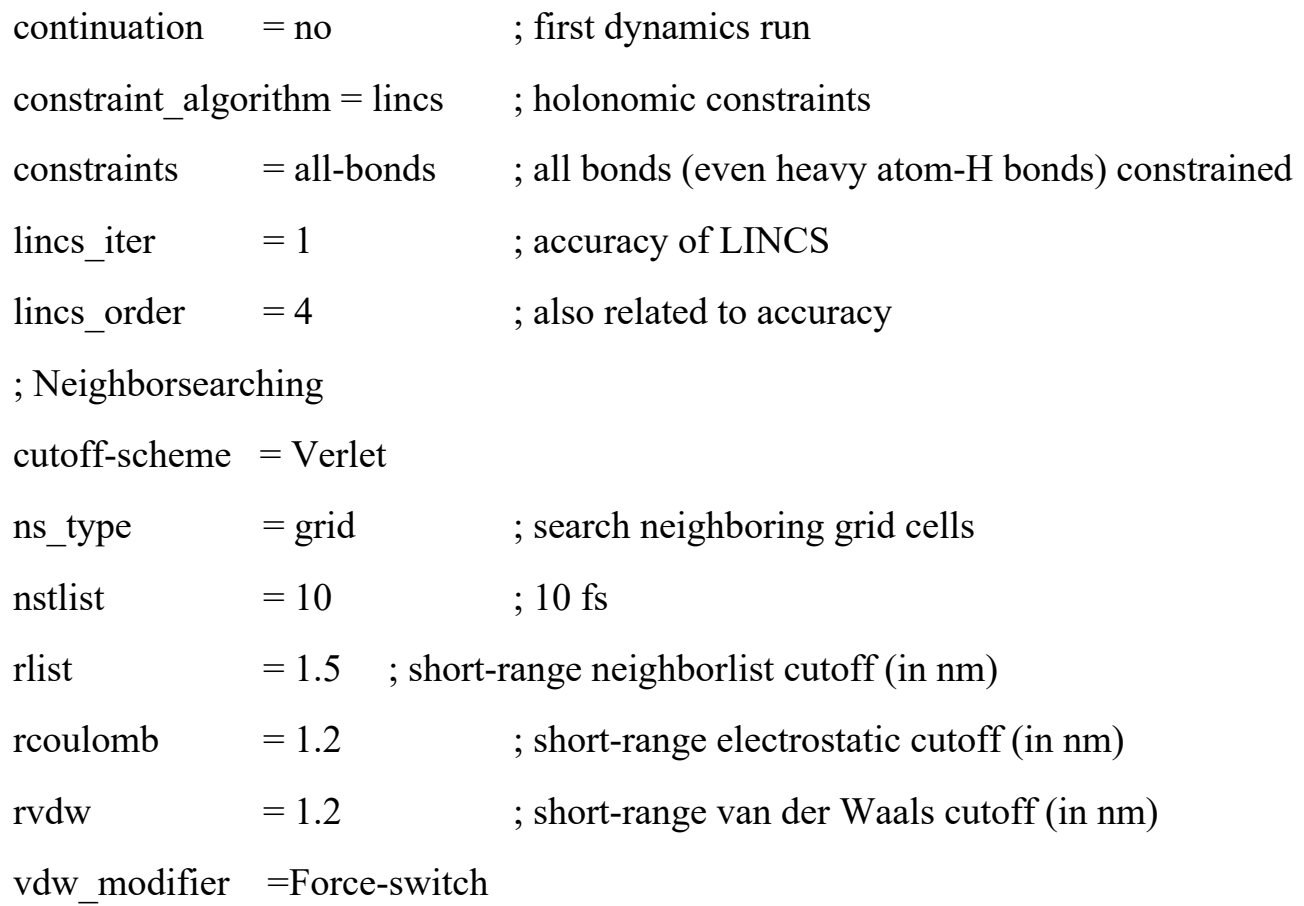




$$
\begin{aligned}
& \text { vdwtype = Cut-off } \\
& \text { coulombtype }=\text { PME-Switch } \\
& \text { rcoulomb_switch }=1.15 \\
& \text { rvdw-switch }=1.15 \\
& \text { verlet-buffer-tolerance }=-1
\end{aligned}
$$

\section{; Electrostatics}

pme_order $\quad=4 \quad$; cubic interpolation

fourierspacing $=0.16 \quad$; grid spacing for FFT

; Temperature coupling is on

annealing $=$ single

annealing-npoints $=11$

annealing-time $=01002003004005006007008009001000$

annealing-temp $=538.15518 .15498 .15478 .15458 .15438 .15418 .15398 .15378 .15358 .15338 .15$

tcoupl $\quad=$ V-rescale $\quad$ modified Berendsen thermostat

tc-grps $\quad=$ system $\quad$; two coupling groups - more accurate

tau_t $\quad=0.1 \quad ;$ time constant, in ps

ref_t $\mathrm{t} \quad$; reference temperature, one for each group, in $\mathrm{K}$

; Pressure coupling is off

pcoupl $\quad=$ no $\quad ;$ no pressure coupling in NVT

; Periodic boundary conditions

pbc $\quad=x y z \quad ; 3-D$ PBC

; Dispersion correction

DispCorr $\quad=$ EnerPres $\quad$; account for cut-off vdW scheme

; Velocity generation

$\begin{array}{llr}\text { gen_vel } & =\text { yes } & ; \text { assign velocities from Maxwell distribution } \\ \text { gen_temp } & =338.15 & ; \text { temperature for Maxwell distribution } \\ \text { gen_seed } & =-1 & ; \text { generate a random seed }\end{array}$




\section{NPT to obtain the density}

; Run parameters

$\begin{array}{lll}\text { integrator } & =\mathrm{sd} & ; \text { leap-frog integrator } \\ \text { nsteps } & =10000000 & \\ \mathrm{dt} & =0.002 & ; 2 \mathrm{fs}\end{array}$

; Output control

$\begin{array}{ll}\text { nstxout } & =3000 \\ \text { nstvout } & =3000 \\ \text { nstenergy } & =3000 \\ \text { nstlog } & =3000\end{array}$

; Bond parameters

constraint_algorithm $=$ lincs $\quad$; holonomic constraints

constraints $\quad=$ h-bonds $\quad$; all bonds (even heavy atom-H bonds) constrained

lincs_iter $\quad=1 \quad$; accuracy of LINCS

lincs_order $=4 \quad$; also related to accuracy

; Neighborsearching

\begin{tabular}{|c|c|c|}
\hline ns_type & $=$ grid & ; search neighboring grid cells \\
\hline nstlist & $=5$ & ; $10 \mathrm{fs}$ \\
\hline rlist & $=1.2$ & ; short-range neighborlist cutoff (in nm) \\
\hline rcoulomb & $=1.2$ & ; short-range electrostatic cutoff (in nm) \\
\hline rvdw & $=1.2$ & ; short-range van der Waals cutoff (in nm) \\
\hline \multicolumn{3}{|l|}{; Electrostatics } \\
\hline coulombtype & $=\mathrm{PME}$ & ; Particle Mesh Ewald for long-range electrostatics \\
\hline pme_order & $=4$ & ; cubic interpolation \\
\hline fourierspacing & $=0.16$ & ; grid spacing for FFT \\
\hline \multicolumn{3}{|c|}{; Temperature coupling is on } \\
\hline tcoupl & $=\mathrm{V}$-rescale & ; modified Berendsen thermostat \\
\hline tc-grps & $=$ system & ; two coupling groups - more accurate \\
\hline tau_t & $=0.1$ & ; time constant, in ps \\
\hline ref_t & $=338.15$ & ; reference temperature, one for each group, in $\mathrm{K}$ \\
\hline
\end{tabular}

; Pressure coupling is on 
pcoupl $=$ Parrinello-Rahman ; Pressure coupling on in NPT

pcoupltype = isotropic ; uniform scaling of box vectors

tau $\_$p $\quad$; time constant, in ps

ref $\_$p $\quad=1.0 \quad$; reference pressure, in bar

compressibility $=4.5 \mathrm{e}-5$; isothermal compressibility of water, bar ${ }^{\wedge}-1$

refcoord_scaling $=$ com

; Periodic boundary conditions

$\mathrm{pbc} \quad=\mathrm{xyz} \quad ; 3-\mathrm{D}$ PBC

; Dispersion correction

DispCorr $\quad=$ EnerPres $\quad$; account for cut-off vdW scheme

; Velocity generation

gen_vel $\quad$ no $\quad$; Velocity generation is off

\section{Equilibration}

; Run parameters

$\begin{array}{lll}\text { integrator } & =\mathrm{sd} & \\ \text { nsteps } & =5000000 & \\ \mathrm{dt} & =0.002 & ; 2 \mathrm{fs}\end{array}$

; Output control

nstxout $\quad=3000$

nstvout $\quad=3000$

nstenergy $=3000$

nstlog $\quad=3000$

; Bond parameters

continuation $=$ no ; first dynamics run

constraint_algorithm $=$ lincs $\quad$; holonomic constraints

constraints = all-bonds ; all bonds (even heavy atom-H bonds) constrained

lincs_iter $\quad=1 \quad$; accuracy of LINCS

lincs_order $=4 \quad$; also related to accuracy

; Neighborsearching 


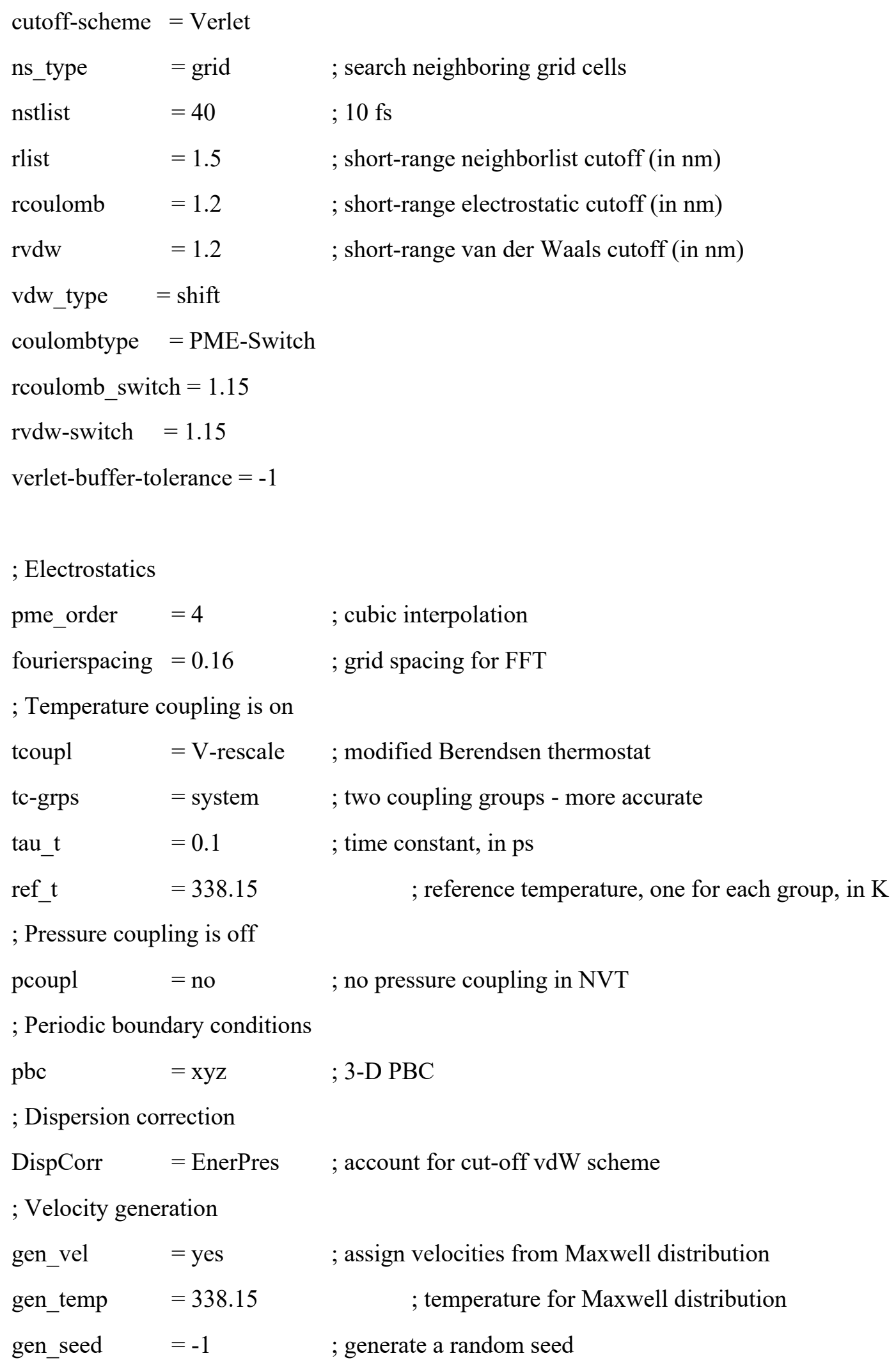


NVE

; Run parameters

$\begin{array}{lll}\text { integrator } & =\mathrm{sd} & ; \text { leap-frog integrator } \\ \mathrm{nsteps} & =175000000 & \\ \mathrm{dt} & =0.002 & ; 2 \mathrm{fs}\end{array}$

; Output control

nstxout $\quad=10000$

nstvout $\quad=10000$

nstenergy $\quad=10000$

nstlog $=10000$

; Bond parameters

;continuation $=$ no $\quad$; first dynamics run

;constraint_algorithm $=$ lincs $;$ holonomic constraints

; constraints $=$ h-bonds ; all bonds (even heavy atom-H bonds) constrained

;lincs_iter $=1 \quad$; accuracy of LINCS

;lincs_order $=4 \quad$; also related to accuracy

; Neighborsearching

cutoff-scheme $=$ Verlet

ns_type $\quad$ grid $\quad$; search neighboring grid cells

nstlist $\quad=40 \quad ; 10 \mathrm{fs}$

rlist $\quad=1.5 \quad$; short-range neighborlist cutoff (in $\mathrm{nm}$ )

rcoulomb $\quad=1.2 \quad$; short-range electrostatic cutoff (in nm)

rvdw $\quad=1.2 \quad ;$ short-range van der Waals cutoff (in nm)

vdw_type $\quad=$ shift

coulombtype = PME-Switch

rcoulomb_switch $=1.15$

rvdw-switch $=1.15$

verlet-buffer-tolerance $=-1$

; Electrostatics 
pme_order $=4 \quad$; cubic interpolation

fourierspacing $=0.10 \quad$; grid spacing for FFT

; Temperature coupling is on

; Pressure coupling is off

pcoupl $\quad=$ no $\quad$; no pressure coupling in NVT

; Periodic boundary conditions

$\mathrm{pbc} \quad=\mathrm{xyz} \quad ; 3-\mathrm{D}$ PBC

; Dispersion correction

DispCorr $\quad=$ EnerPres $\quad$; account for cut-off vdW scheme 